\title{
Infantile masturbation mimicking paroxysmal disorders
}

\author{
Hassib Narchi
}

Pediatric Department Sandwell General Hospital, West Bromwich,United Kingdom

\begin{abstract}
Three girls, aged between 7 months and 5 years, were referred with recurrent paroxysmal manifestations since the age of 6 to 10 months. All three had an entirely normal clinical examination, were initially thought to have epileptic fits, their electroencephalogram was unremarkable, and the 'fits' remained refractory to anticonvulsant therapy. They all had stereotypical and reproducible manifestations, with clear evidence of distractibility. Videotaping the manifestations by parents was crucial for the diagnosis. All three were diagnosed to have autostimulatory behaviour or infantile masturbation. No pathological psychosocial issues were identified in any of the families who accepted the diagnosis with great relief. Infantile masturbation mimics common pediatric problems, and, if unrecognized, may lead to considerable parental anxiety, unnecessary investigations and inappropriate and potentially harmful therapy. (J Pediatr Neurol 2003; 1(1): 43-45).
\end{abstract}

Key words: masturbation, self stimulation, behaviour, nfant, child.

Correspondence: Hassib Narchi, M.D.,

Consultant Pediatrician, Pediatric Department Sandwell, General Hospital West Bromwich,

B71 4HJ United Kingdom.

Tel: (44) 121607 6417, fax: (44) 1216073596

E-mail: hassibnarchi@hotmail.com

Received: April 14, 2003

Revised: May 7, 2003.

Accepted: May 8, 2003.

\section{Introduction}

Recurrent paroxysmal manifestations in childhood have a wide differential diagnosis, with neurologic and cardiac causes ranking high on the list for the family and the treating physician. Proper diagnosis is essential, appropriate investigations are needed and appropriate specific therapy may be required. Infantile masturbation is not always recognizable and therefore not readily included in that differential diagnosis, leading therefore to unnecessary investigations and treatment. We report three girls ( 7 months to 5 years) with infantile masturbation who were thought to have epileptic seizures, leading to considerable parental anxiety, unnecessary investigations and inappropriate and potentially harmful therapy.

\section{Case Reports \\ Case 1}

A 5-year-old girl presented with recurrent episodes of stiffening since she was 10 months old. While playing or watching television, she would kneel down, thighs adducted, rocks her body forward and backward, her trunk stiffens and she stares into space. No clonic movements, colour changes, eyes rolling or incontinence are reported during these episodes, and she stops as soon as her parents tell her to do so. The frequency of these episodes is very variable and she is entirely asymptomatic in-between. A hyperventilation test did not induce any absences. Following a normal electroencephalogram (EEG), she was started on valproate therapy without much success.

\section{Case 2}

A 7-month-old female infant had a recent onset of repetitive bouts of stiffening of the body, hyperextension and adduction of hips, grunting and irregular breathing, with the face becoming red and sweaty. Each episode may last 1 to 15 
minutes and may occur several times a day. The child is well immediately after and is asymptomatic between these episodes. Serum biochemistry, cranial ultrasound and EEG were normal and the family was reluctant to have antiepileptic therapy started.

\section{Case 3}

Since age of 6 months a one-year old girl was having up to 40 daily paroxysmal episodes. Each episode occurs only when she is either lying on her front, or on her knees. She crouches down, pelvis pressed on the floor, lifts herself onto her extended arms, looks up, arches her back, throws her head back, her 1 egs shake, and she goes stiff. Her face is strained as if frightened, sometimes dribbles, he looks vacant, stares like if "in a trance", her eyes are glazed but continue to move erratically. Then she suddenly becomes floppy and goes into a sleep. During such an episode, she immediately "comes round" when spoken to or if told to stop. Sometimes, as if she seems to realise when an "attack" is about to happen, she runs to her mother, appears frightened, hangs on to her mother, grips her clothes intermittently, looks vacant, then goes floppy and sleeps for few minutes. Characteristically, these episodes occur only when tired or bored, and never when she is playing or distracted. When admitted to hospital for observation, her mother expressed the wish to observe her when in a cublicle as she was certain that nothing would happen if she was observed in the open ward where she would be distracted and playing with other children. Serum biochemistry and metabolic studies were normal. She had a normal cranial computed tomograhy scan and an EEG was normal even during one of these episodes.There was no response to carbamazepine or valproate therapy.

All 3 girls had a normal development and an entirely normal physical examination. No pathological psychosocial issues were identified in any of the families. The parents were asked to videotape these episodes at home. Review of the tapes showed characteristic findings: the manifestations were always stereotypical, reproducible, with clear evidence of voluntary control and distractibility. All these episodes were remarkably similar to what has been previously reported in self-stimulatory behaviour or infantile masturbation. This diagnosis was explained to the parents and the families accepted the diagnosis with great relief.

\section{Discussion}

The diagnosis of masturbation is difficult in young children, especially girls and this may lead to unnecessary investigations and treatment (1). This condition occurs at any age, the youngest reported child being 2 months old, but has also been reported in a male fetus by ultrasound $(1,2)$. A minimal perineal irritative focus with discomfort, such as dermatitis, urinary tract infection, pinworms or constipation, may precede the manifestations. Stressful events such as weaning, birth of a sibling or separation from the parents may also be precipitating factors (3). Like other self-stimulatory behaviours, masturbation tends to occur during boredom, loneliness, anxiety or excitement.

In young girls, tightening of the thighs, rocking pelvic movements or other rhythmic activities, mechanical pressure application to suprapubic area, accompanied by grunting, facial flushing, irregular breathing, and sweating may occur and may be misinterpreted as abdominal pain or urinary symptoms (1). The association with a vacant and glassy stare, associated with rigidity, grunting or rocking movements may suggest epileptic seizures (1). These episodes may last from a minute to several hours, there is no alteration of consciousness and the child usually stops this activity when told. The physical examination is usually normal or may show mild perineal irritation. Excluding local irritative causes in the perineum should be undertaken and the possibility of child sexual abuse should always be looked for (4).

A detailed history is essential and personal observation of these episodes helps to confirm the diagnosis. Videotaping the episodes by the parents is also very helpful for the diagnosis and also helps with the management of this condition (1).

Spontaneous resolution is the norm and in $78 \%$ of the cases this occurs within two years. Improvement may be delayed in younger patients, those who begin to masturbate earlier and those who masturbate more frequently (3).

It is more helpful and certainly more acceptable to the majority of families to use the term autostimulation or habit rather than masturbation when discussing this entity (5). Management consists of explaining to the parents the harmless and painless nature of the autostimulatory activity, aiming for decreasing the reinforcing effect of any of their inappropriate responses to the condition. For example, punishment tends to reinforce the behaviour and should therefore be discouraged (6).

Masturbation is a normal and common behaviour in young children and may mimic common pediatric conditions. Failure to recognize it may lead to considerable parental anxiety, unnecessary investigations and inappropriate and potentially harmful therapy. Health care workers should be familiar with this benign condition and should always include it in the differential diagnosis of the common pediatric conditions it may mimic. 


\section{References}

1. Fleisher DR, Morrison A. Masturbation mimicking abdominal pain or seizures in young girls. J Pediatr 1990; 116: 810-814.

2. Meizner I. Sonographic observation of in utero fetal "masturbation". J Ultrasound Med 1987; 6: 111.

3. Ünal F. The clinical outcome of childhood masturbation. Turk J Pediatr 2000; 42: 304-307.
4. Sauzier M. Disclosure of child sexual abuse. For better or worse. Psychiatr Clin North Am 1989; 12: 455-469.

5. Green M. Interviewing. In: Green M, Haggerty R (eds). Ambulatory Pediatrics. Vol. 2. Philadelphia: WB Saunders, 1977, pp 441-453.

6. Gallo AM. Early childhood masturbation: a developmental approach. Pediatr Nurs 1979; 5: 4749. 